\title{
Identical twins with papilloedema and cranial nerve palsies
}

\author{
A. J. CORBETT A ND M. POLLOCK \\ From the Neurology Section, Department of Medicine, University of Otago Medical School, \\ Dunedin, New Zealand
}

S UMMARY Central nervous system involvement has not been reported previously as a manifestation of sarcoidosis in monozygotic twins. Twin sisters developed multiple cranial nerve palsies and papilloedema within a two month period. Clinical features and the course of their diseases were remarkably similar.

The familial occurrence of sarcoidosis is well documented (Sharma et al., 1976). Thus Selroos et al. (1973) reviewed the concomitant occurrence of sarcoidosis in 13 identical twins. There is no previous report of central nervous system involvement by sarcoidosis in twins, although isolated facial nerve palsy with parotid gland swelling has been documented (Sherer and Kelly, 1949).

\section{Case reports}

Monozygotic twin sisters aged 27 years developed cranial nerve palsies and papilloedema within a two month period. The first twin sister was seen with erythema nodosum in June 1975. Six weeks later she developed over a few days a dense left facial nerve palsy. Three weeks later she was admitted to hospital with severe frontal headache, blurring of vision, intermittent horizontal diplopia, and vertigo. Examination revealed bilateral papilloedema, a right lateral rectus palsy, and a mild left facial nerve palsy. There were no palpable lymph nodes. Investigations included normal chest and skull radiographs and a normal isotope brain scan. The ESR was $58 \mathrm{~mm} / \mathrm{hr}$ on admission, rising to $73 \mathrm{~mm} / \mathrm{hr}$ after three days, but the blood screen was normal. The EEG showed bilateral slowing, more marked on the right. A right carotid angiogram demonstrated slowing of the internal carotid circulation consistent with raised intracranial pressure but no other abnormality. A

Address for reprint requests: Associate Professor M. Pollock, Department of Medicine, University of Otago Medical School, PO Box 913, Dunedin, New Zealand.

Accepted 6 February 1979 pneumoencephalogram was normal. The CSF contained one lymphocyte per $\mathrm{mm}^{3}$, protein $0.7 \mathrm{~g} / 1$, and glucose $2.8 \mathrm{mmol} / \mathrm{l}$. She was treated with prednisone $40 \mathrm{mg}$ daily for one month with resolution of her cranial nerve palsies and papilloedema-

Three months later her chest radiograph showee enlarged paratracheal lymph nodes on the right and tomography demonstrated enlarged hilar, sube carinal, and paratracheal lymph nodes. Lung voo umes were slightly reduced with a moderat 8 reduction in single breath carbon monoxide dip fusing capacity. The patient underwent medic astinoscopy for biopsy of a paratracheal lymp? node. Histological examination demonstrated noncaseating granulomata characteristic of sarcoidosis. She received no further steroid therapy.

Her radiographic abnormalities resolved during the next six months, and she has remained symptom-free over a further 18 month review period.

The second sister developed right facial nerve palsy two months after her twin, associated with numbness of the right side of her face and tongue, and abnormal taste sensation. Examination showed bilateral papilloedema with an enlarged blind spot on the right, reduced sensation over the right trigeminal nerve territory, and a partial right facial nerve weakness with impaired taste sensation over the right anterior two-thirds of the tongue. Investigations included normal chest and skull radiographs, isotope brain scan, and EEG Blood screen and ESR were normal. The CSF contained 102 white blood cells per $\mathrm{mm}^{3}$, predominantly granulocytes, protein $0.64 \mathrm{~g} / 1$, and glucose $2.5 \mathrm{mmol} / \mathrm{l}$. She was treated with prednisone $40 \mathrm{mg}$ daily for one month. 
Papilloedema and cranial nerve palsies resolved in two months, and she has remained symptomfree for 18 months.

Blood and histocompatibility antigen typing have confirmed that the twins are monozygotic.

\section{Discussion}

Although not conclusively proved, it is reasonable to hypothesise that both twins suffered from neurosarcoidosis. In the absence of evidence of coexisting involvement of other organs, no tissue diagnosis was achieved in the second twin.

The premortem diagnosis of sarcoidosis of the central nervous system may prove difficult. Neurological dysfunction is frequently the only clinical manifestation of sarcoidosis at presentation (Delaney, 1977). Random tissue biopsy, especially of the liver or scalene fat pad, may establish a diagnosis when the neurological features are the only indication of the site of the disease. The Kveim test takes four to six weeks and is influenced by steroid therapy, thus limiting its value. Even brain needle biopsy may not be helpful since the granulomata are frequently widely dispersed and usually predominate at the base of the brain. The most consistent findings on CSF examination are raised protein, normal or low glucose, and a variable lymphocytic pleocytosis. Cytology, culture for bacteria, acid-fast bacilli, and fungi are negative. However the CSF may remain normal even at the height of the disease (Douglas and Maloney, 1973).

Involvement of the central nervous system by sarcoidosis is seen in only about $5 \%$ of cases (Sharma, 1975). Sarcoid granulomata are most commonly found in the meninges causing nodular masses or, more commonly, an adhesive meningitis. The latter results in cranial neuropathies, with facial palsies the most frequent manifestation (Silverstein et al., 1965). Much less frequent are sarcoid granulomata forming tumour-like masses within the brain parenchyma. Papilloedema may result from obstruction to CSF flow, mass effect, or optic nerve involvement (Blain et al., 1965). There is no constant association of signs and symptoms which can be grouped to describe a characteristic syndrome of cerebral sarcoidosis. Computerised tomography (CAT) is the most informative neuroradiological study with the granulomata showing as high attenuation lesions homogeneously enhancing with little surrounding oedema. The CAT scan was positive in $77 \%$ of cases of neurosarcoidosis (Kendall and Taylor, 1978). With availability of CAT scanning there remain few indications for angiography or air studies.
Occasionally angiography may differentiate an avascular granuloma from a tumour with pathological circulation. Similarly, in the presence of chiasmal involvement or cranial nerve palsies air study may at times demonstrate small lesions not shown by CAT scanning.

Corticosteroid therapy provides the only possibility of influencing the outcome of this disease favourably, and treatment may have to be started before a definitive diagnosis has been made. It is difficult to assess the effectiveness of steroids but, in a worldwide investigation, Siltzbach et al. (1974) considered neurosarcoidosis an absolute indication for steroid therapy.

The familial occurrence of sarcoidosis is well recognised, and multigenic and autosomal recessive modes of inheritance have been proposed by Headings et al. (1976). It is more frequent in monozygotic than dizygotic twins (British Thoracic and Tuberculosis Association, 1973). In 13 pairs of identical twins affected by sarcoidosis the disease manifestations and course tended to be remarkably similar in each twin pair (Selroos $e t$ al., 1973).

The stereotyped presentation in familial sarcoidosis where there is a tendency for sarcoidosis to develop at the same age and with similar clinical features (Sharma et al., 1976) may indicate a constitutional factor predisposing to reactivity to some unknown agent. Our twins presented almost simultaneously with an uncommon manifestation of sarcoidosis not previously reported in monozygotic twins. The twins followed similar courses with rapid and complete resolution of their disease.

\section{References}

Blain, J. G., Riley, W., and Logothetis, J. (1965). Optic nerve manifestations of sarcoidosis. Archives of Neurology (Chicago), 13, 307-309.

British Thoracic and Tuberculosis Association (1973). Familial associations in sarcoidosis. Tubercle, 54, 87-98.

Delaney, P. (1977). Neurological manifestations of sarcoidosis. Annals of Internal Medicine, 87, 336-345.

Douglas, A. C., and Maloney, A. F. J. (1973). Sarcoidosis of the central nervous system. Journal of Neurology, Neurosurgery, and Psychiatry, 36, 1024 1033.

Headings, V. E., Weston, D., Young, R. C., and Hackney, R. L. (1976). Familial sarcoidosis with multiple occurrences in eleven families: a possible mechanism of inheritance. Annals of the New York Academy of Sciences, 278, 377-385.

Kendall, B. E., and Taylor, G. L. V. (1978). Radiological findings in neurosarcoidosis. British Journal of Radiology, 51, 81-92. 
Selroos, O., Sellergren, T-L., Vuorio, M., and Virolainen, M. (1973). Sarcoidosis in identical twins. American Review of Respiratory Disease, 108, 14011406.

Sharma, O. P. (1975). Sarcoidosis, a Clinical A pproach, pp. 97-102. Charles C. Thomas: Springfield, Illinois.

Sharma, O. P., Neville, E., Walker, A. N., and James, D. G. (1976). Familial sarcoidosis: a possible genetic influence. Annals of the New York Academy of
Sciences, 278, 386-399.

Sherer, J. F., and Kelly, R. T. (1949). Sarcoidosis in identical twins. New England Journal of Medicine, 240, 328-330.

Siltzbach, L. E., James, D. G., and Neville, E. (1974). Course and prognosis of sarcoidosis around the world. American Journal of Medicine, 57, 847-852. Silverstein, A., Feuer, M. M., and Siltzbach, L. E. (1965). Neurologic sarcoidosis. Archives of Neurology (Chicago), 12, 1-11. 